\title{
Line reconstruction using prior knowledge in single non-central view
}

\author{
Jesus Bermudez-Cameo ${ }^{1}$ \\ bermudez@unizar.es \\ Cédric Demonceaux ${ }^{2}$ \\ cedric.demonceaux@u-bourgogne.fr \\ Gonzalo Lopez-Nicolas ${ }^{1}$ \\ gonlopez@unizar.es \\ José J. Guerrero \\ josechu.guerrero@unizar.es
}

${ }^{1}$ Instituto de Investigación en Ingeniería de Aragón (I3A)

Universidad de Zaragoza

Zaragoza, Spain

${ }^{2}$ Le2i - Institut Universitaire de Technologie Le Creusot

Université de Bourgogne

Le Creusot, France

Line projections in non-central systems contain more geometric information than central systems. The four degrees of freedom of the 3D line are mapped to the line-image and the 3D line can be theoretically recovered from 4 projecting rays (i.e. line-image points) from a single non-central view [3]. If the non-central system is properly calibrated we obtain a metric reconstruction of the 3D line. In practice, extraction of line-images is considerably more difficult and the resulting reconstruction is imprecise and sensitive to noise.

In this paper we explore the reconstruction accuracy improvements when we impose geometrical constraints [1] exploiting prior knowledge. In particular, when the lines of the scene are arranged in two orthogonal directions and we know prior information about the direction of one of this directions (typically the vertical direction), the complexity of line fitting reduces, the accuracy of the metric reconstruction improves, and the extraction procedure is simplified.

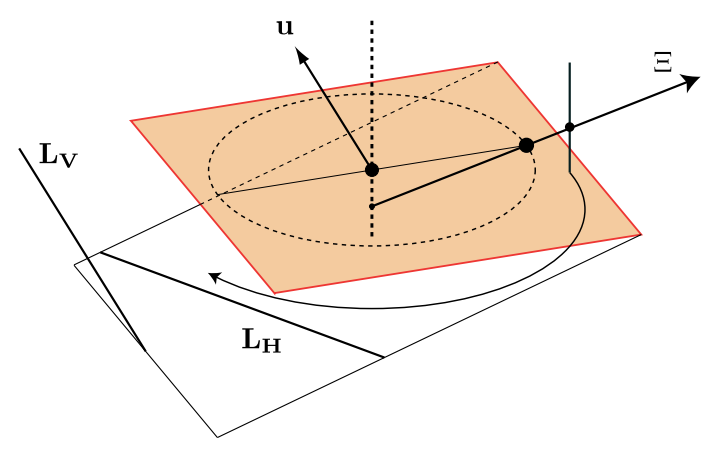

Figure 1: Consider a Manhattan setting with horizontal lines $\mathbf{L}_{H}$ and vertical lines $\mathbf{L}_{V}$. A non-central circular panoramic system is considered in unknown position and orientation but the vertical direction $\mathbf{u}$ of lines $\mathbf{L}_{V}$ in its own reference system is known.

The first restriction considers a 3D line parallel to a plane, and takes advantage of the prior knowledge of the vertical direction to fit horizontal lines. In this case only three rays are needed to fit the 3D line. Using the geometrical properties of Plücker description of lines it is possible to define the intersection operator among the sought line $\mathbf{L}$ and the three projection rays $\Xi_{k}$ obtaining a linear subspace of dimension two contained in the projective space $\mathbb{P}^{5}$.

$$
\mathbf{L}=\mathbf{L}_{0}+\mathbf{L}_{1} \lambda_{1}+\mathbf{L}_{2} \lambda_{2} .
$$

By imposing that the line must be orthogonal to the vertical direction $\mathbf{u}$ and the solution must be a line (Plücker constraint) we obtain a closed form solution for the parameters $\lambda_{1}$ and $\lambda_{2}$. This is obtained by solving the quadratic equation

$$
\begin{array}{r}
b_{1} \lambda_{1}^{2}+2 b_{2} \lambda_{1}+b_{3}=0 \quad \text { where } \\
b_{1}=\beta_{2211}-2 \beta_{1212}+\beta_{1122} \\
b_{2}=\beta_{0122}-\beta_{0212}-\beta_{1202}+\beta_{2201} \\
b_{3}=\beta_{2200}-2 \beta_{0202}+\beta_{0022}
\end{array}
$$

and $\beta_{i j k l}=\mathbf{L}_{i}^{\top}{ }^{\top} \mathbf{L}_{j} \mathbf{u}^{\top} \mathbf{l}_{k} \mathbf{u}^{\top} \mathbf{l}_{l}$ for $\lambda$ and computing the corresponding line

$$
\mathbf{L}=\left(\mathbf{u}^{\top} \mathbf{l}_{2} \mathbf{L}_{0}-\mathbf{u}^{\top} \mathbf{l}_{0} \mathbf{L}_{2}\right)+\left(\mathbf{u}^{\top} \mathbf{l}_{2} \mathbf{L}_{1}-\mathbf{u}^{\top} \mathbf{l}_{1} \mathbf{L}_{2}\right) \lambda_{1}
$$

The second restriction considers a 3D line with known direction $\mathbf{l}$, and again takes advantage of the prior knowledge of the vertical direction for fitting vertical lines. In this case the solution for the momentum vector of the line $\mathbf{I}$ is a closed form expressed as

$$
\overline{\mathbf{l}}=\left(\begin{array}{c}
\xi_{1}^{\top} \\
\xi_{2}^{\top} \\
\mathbf{l}^{\top}
\end{array}\right)^{-1}\left(\begin{array}{c}
-\bar{\xi}_{1}^{\top} \mathbf{l} \\
-\bar{\xi}_{2}^{\top} \mathbf{l} \\
0
\end{array}\right)
$$

Both formulations are integrated in a line-extraction pipeline, which is tested with synthetic and real non-central circular panoramas [2].

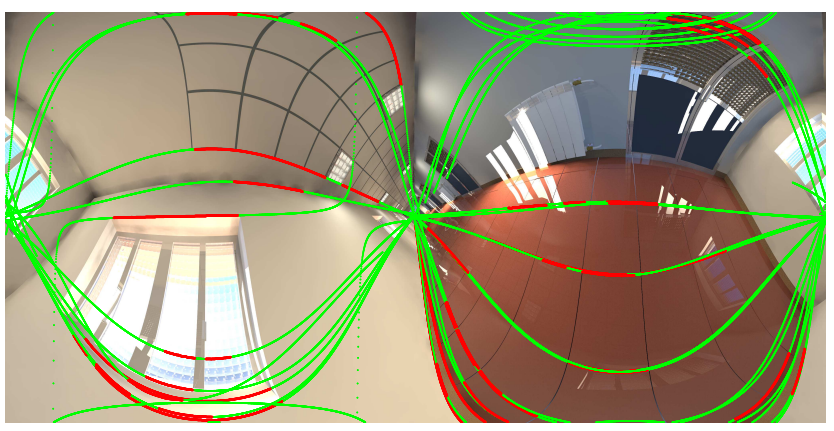

(a)

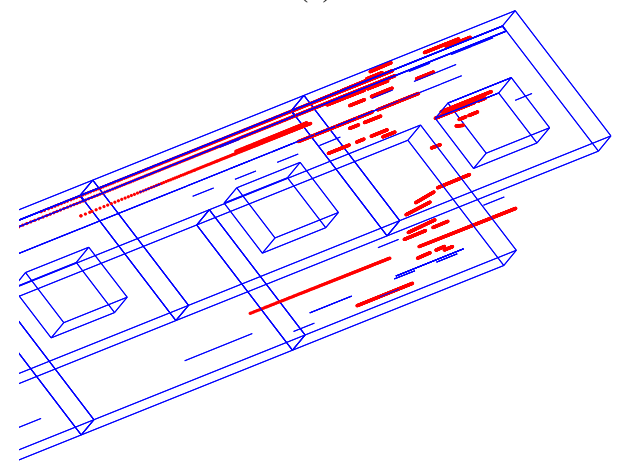

(b)

Figure 2: Extraction and reconstruction example from synthetic single non-central panorama: (a) Extracted lines following the main direction over a non-central panorama (green for the parametric line and red for supporting points). (b) 3D view of the ground truth (in blue) and the reconstruction of the 3D line segments (in red).

In addition, we evaluate the performance of the robust extractor and the accuracy of the proposal in comparison with the unconstrained method. We conclude that the proposal outperforms the unconstrained algorithm and provides good results taking into account typical accuracy of standard commercial IMUs (error around $0.5 \mathrm{deg}$ ).

[1] Jesus Bermudez-Cameo, Joao P. Barreto, Gonzalo Lopez-Nicolas, and Jose. J Guerrero. Minimal solution for computing pairs of lines in non-central cameras. In The 12th Asian Conference on Computer Vision (ACCV), 2014.

[2] Marc Menem and Tomás Pajdla. Constraints on perspective images and circular panoramas. In British Machine Vision Conference (BMVC), pages 1-10, 2004.

[3] Seth Teller and Michael Hohmeyer. Determining the lines through four lines. Journal of graphics tools, 4(3):11-22, 1999. 